\title{
Die Eliteschule des Fußballs: eine Längsschnittstudie in stresstheoretischer Perspektive zur Verbindung von Schule und Leistungssport
}

\author{
Jennifer Breithecker, Hans Peter Brandl-Bredenbeck
}

\section{Einleitung und Problemstellung}

Um leistungssportliche Karrieren gezielt und nachhaltig $\mathrm{zu}$ fördern, wurden seit den 1990er Jahren verschiedene Verbundsysteme wie die Sportbetonte Schule, die Partnerschule des Leistungssports, die NRW-Sportschule oder die Eliteschule des Sports eingerichtet (Breithecker 2016). Ziel dieser Fördermodelle ist es, sportartenübergreifend junge NachwuchsathletInnen sowohl in ihrem schulischen als auch in ihrem sportlichen Karriereweg zu unterstützen. In ihrem Grundsatz versuchen alle Einrichtungen durch strukturelle Arrangements und individuelle Abstimmung mit den AthletInnen eine Verzahnung von Schulleben, Verein und Internat zu gewährleisten, um so die Doppelbelastung aus Schule und Leistungssport auszubalancieren sowie eine gesunde Persönlichkeitsentwicklung der Heranwachsenden sicherzustellen. Seit dem Jahr 2006 hat darüber hinaus der Deutsche Fußballbund (DFB) durch die Einrichtung von Eliteschulen des Fußballs ein sportartenspezifisches, nationales Unterstützungsmodell etabliert. Eliteschulen des Fußballs sind an ein nahgelegenes Fußballleistungszentrum gekoppelt und müssen alle Schulformen einschließen (DFB 2016). Zu den konkreten Unterstützungsmaßnahmen zählen u. a. die Einrichtung von Sportprofilen (z. B. Sportkurse, Sportzüge), Hausaufgabenbetreuung, Nachhilfe, zeitlich flexible Klausurtermine und zusätzliche Trainingseinheiten am Vormittag (ebd.). Der DFB (o. D., S. 14) versteht seine Einrichtung als „Eckpfeiler“ für eine ganzheitliche Förderung unter Ausbildung einer starken Persönlichkeit. $\mathrm{Ob}$ und inwieweit er diesem Auftrag gerecht wird, soll Gegenstand des vorliegenden Beitrags sein.

\section{Forschungsstand und Untersuchungsfragen}

Im Kontext der Doppelbelastungsforschung hat sich in der Sportpädagogik in den letzten Jahren ein stresstheoretischer Ansatz unter Bezug zur klassischen, kognitivtransaktionalen Stresstheorie nach Lazarus (Lazarus/Folkman 1984) durchgesetzt. Dabei ist der Grad der Stressbelastung eines Individuums einerseits von den wahrgenommenen Anforderungen und andererseits von der Reaktionskapazität unter Rückgriff auf soziale und personale Ressourcen abhängig. Entsprechend dieser subjektiven Bewertungsprozes- 
se kommt es zu individuellen Copingversuchen, die Anpassungsleistungen nach sich ziehen und so das psycho-physische Wohlbefinden stabilisieren (Faltermeier 1987). Als soziale Ressourcen werden Personen aus dem direkten sozialen Nahraum von Heranwachsenden wie Eltern, Freunde bzw. Freundinnen, LehrerInnen und KlassenkameradInnen verstanden (Wild/Möller 2015). Zu den personalen Ressourcen gehören individuelle Kontroll- bzw. Kompetenzüberzeugungen sowie sozialkognitive Strukturniveaus (Schwarzer/Jerusalem 2002). Gestärkt durch diese Ressourcen oder Schutzfaktoren können Heranwachsende erfolgreich mit belastenden Lebensumständen und den negativen Folgen von Stress umgehen (Hemming 2015). Im Kontext der Doppelbelastung aus Schule und Leistungssport wird die Ausbildung dieser schützenden Ressourcen allerdings ambivalent betrachtet: Einerseits könnte ein Engagement im Leistungsfußball durch hohe insbesondere schulische und zeitliche Anforderungen zu einer Abnahme sozialer und personaler Ressourcen führen, andererseits birgt das leistungssportliche Engagement die Möglichkeit, durch individuelle Kompetenzerfahrung und soziale Netzwerke besonders viele Schutzfaktoren auszubilden (Güllich/Richartz 2016).

Die Erforschung dieses Spannungsfeldes insgesamt war bereits Gegenstand diverser sportwissenschaftlicher Untersuchungen. Grob lassen sich diese Forschungsbemühungen in drei verschiedene Kategorien unterteilen:

(1) Qualitative und quantitative Studien im Quer- und Längsschnitt außerhalb von Verbundsystemen (Hemming 2015; Richartz 2000; Richartz/Brettschneider 1996; Richartz/ Hoffmann/Sallen 2009).

(2) Quantitative Längsschnittstudien im Kontext von Verbundsystemen wie die Studie zur Umwandlung der ehemaligen Kinder- und Jugendsportschulen (Ost-)Berlins in Sportbetonte Schulen (Brettschneider/Klimek 2009; Heim 2002), die Evaluation einer Potsdamer Eliteschule des Sports (Beckmann/Szymanski/Elbe/Ehrenspiel 2006) und die Begleituntersuchung einer NRW-Sportschule (Breithecker 2016; Kehne/Breithecker 2017).

(3) Quantitative vereinsbasierende und damit verstärkt breitensportlich orientierte Längsschnittstudien im Bereich des Jugendsports (Brettschneider/Kleine 2002; Gerlach/Brettschneider 2013).

Alle Studien können, trotz hoher Freizeitbelastungen leistungssportlich engagierter Heranwachsender, einen besonders hoch ausgeprägten sowohl sozialen als auch personalen Ressourcenpool junger AthletInnen ausmachen. Insgesamt lassen sich, auch unter Berücksichtigung der Schulnoten, keinerlei Nachteile in Anbetracht der Belastungen aus Schule und Leistungssport nachweisen (Güllich/Richartz 2016). Allerdings handelt es sich dabei überwiegend um sportartübergreifende Forschungsbefunde. Studienergebnisse zur Eliteschule des Fußballs stehen gänzlich aus. An dieser Stelle möchte der vorliegende Beitrag mit folgender Untersuchungsfrage ansetzen: Wie entwickeln sich Freizeitumfänge, Schulnoten sowie soziale und personale Ressourcen von EliteschülerInnen des Fußballs im Vergleich zu RegelschülerInnen an einer Eliteschule des Fußballs? 


\section{Methodik}

Seit dem Jahr 2013 wurde eine bayerische Eliteschule des Fußballs (Gymnasium, Realschule, Mittelschule) in einer Längsschnittuntersuchung im Vergleichsgruppendesign ( $\mathrm{t} 1$ 2013, t2-2014, t3-2015) wissenschaftlich begleitet. Der erste Messzeitpunkt wurde zeitgleich mit der Einrichtung einer Sportklasse im Schuljahr 2013/14 in den fünften Jahrgangsstufen durchgeführt. ${ }^{\top}$ In dieser Sportklasse waren die EliteschülerInnen des Fußballs gemeinschaftlich im Ganztagsunterricht zusammengefasst und erhielten zwei Trainingseinheiten an zwei Vormittagen während der Schulzeit an den Trainingsstätten des nahgelegenen Leistungszentrums.

An der Untersuchung nahmen insgesamt 252 SchülerInnen teil, davon waren 55 EliteschülerInnen des Fußballs (EdF; 54 männlich, 1 weiblich) und 197 RegelschülerInnen (Reg.; 127 männlich, 70 weiblich). Die TeilnehmerInnen verteilten sich etwa gleichmäßig auf alle drei Schulformen (Gymnasium: 20 EdF, 66 Reg.; Realschule: 19 EdF, 46 Reg.; Mittelschule: 16 EdF, 85 Reg.). Längsschnittlich konnten über alle Messzeitpunkte, d. h. von der fünften ( $\mathrm{t} 1$ ) bis zur siebten Klasse (t3), 23 Fußballschüler (23 männlich, 0 weiblich) und 89 RegelschülerInnen (50 männlich, 39 weiblich) erfasst werden. Die Untersuchung erfolgte anonym jeweils an zwei Terminen im Dezember innerhalb einer 45minütigen Unterrichtsstunde. Die Teilnahme der SchülerInnen war freiwillig und wurde zuvor durch eine Einverständniserklärung der Eltern bestätigt.

Bei der Untersuchung kamen ein Wochentagebuch und ein Fragebogen zum Einsatz. Das Wochentagebuch zur Erfassung der Freizeitaktivität bestand aus dem Schulstundenplan der SchülerInnen und einem Zeitraster von Montag bis Sonntag im 15-Minuten-Takt. Die SchülerInnen wurden gebeten, weitere regelmäßige Freizeitaktivitäten in das Raster einzutragen. Der Fragebogen diente zur Erfassung der Noten im Bereich Mathematik, Deutsch und Sport sowie der stressrelevanten Ressourcen unter Verwendung validierter Skalen (Brettschneider/Klimek 2009; Gerlach/Brettschneider 2013; Richartz/Hoffmann/ Sallen 2009). Entsprechend dieser Untersuchungsansätze wurden als personale Ressource das Selbstwertgefühl sowie das schulische und sportliche Selbstkonzept und als soziale Ressource die Fürsorglichkeit der Eltern und des Klassenlehrers bzw. der Klassenlehrerin sowie das soziale Selbstkonzept zur Schulklasse erfasst. Sowohl das Wochentagebuch als auch der Fragebogen kamen bereits bei einer Längsschnittstudie im Rahmen der $N R W$ Sportschule zum Einsatz (Breithecker 2016; Kehne/Breithecker 2017).

Die Dateneingabe erfolgte mithilfe des Programms Remark Office OMR ${ }^{\circledR}$ (Version 8,0 ). Bei der Datenauswertung wurden Varianzanalysen mit Messwiederholung (ANOVA MW) zur statistischen Unterschiedsprüfung zwischen EliteschülerInnen des Fußballs und RegelschülerInnen unter Einsatz des Statistikprogramms IBM SPSS® (Version 23,0) angewendet.

\section{Ergebnisse}

Im Folgenden werden die Ergebnisse der Längsschnittstudie zu den einzelnen Untersuchungsbereichen erläutert. Zunächst erfolgt die Darstellung der Befunde zur Freizeitaktivität, gefolgt von den Ergebnissen zu Noten sowie personalen und sozialen Ressourcen. Alle Untersuchungsergebnisse können der nachstehenden Tabelle (Tab. 1) entnommen werden. 


\section{Freizeitaktivität}

Die Ergebnisse zur Freizeitaktivität zeigen, dass die Eliteschüler ${ }^{2}$ des Fußballs, trotz des schulischen Ganztags und dem damit verbundenen Vormittagstraining an zwei Wochentagen während der Schulzeit, signifikant mehr regelmäßigen Aktivitäten außerhalb der Schule nachgehen (Gruppe: $p=, 002 * * / \eta^{2}=, 095$ ). Der Umfang der Freizeitaktivität nimmt dabei insbesondere für die Fußballer von der fünften bis zur siebten Klassenstufe zu (MZPxGruppe: $\left.p=, 018 * * * / \eta^{2}=, 095\right)$. Weiter wird deutlich, dass die Freizeitaktivität der Fußballer im Vergleich zu den RegelschülerInnen überwiegend im sportlichen Bereich zu verorten ist (Gruppe: $p \leq, 001 * * * / \eta^{2}=, 323$ ). Der Umfang sportlicher Aktivität beläuft sich in der fünften Klassenstufe auf 5,23 Stunden, in der sechsten Klassenstufe auf 8,15 Stunden und in der siebten Klassenstufe auf 9,20 Stunden (MZPxGruppe: $\left.p=, 001 * * * / \eta^{2}=, 070\right)$. Die RegelschülerInnen sind ohne schulischen Ganztag mit ca. fünf Stunden pro Woche deutlich weniger in Freizeitaktivitäten außerhalb der Schule eingebunden.

\section{Noten}

Der Blick auf die Schulnoten macht deutlich, dass alle SchülerInnen gleichermaßen durchschnittliche Noten in den Hauptfächern Mathematik und Deutsch aufweisen. Die Mathenoten werden dabei insgesamt von der fünften bis zur siebten Klassenstufe schlechter (MZP: $\left.p=\leq, 001 * * * / \eta^{2}=, 159\right)$. Im Fach Sport erzielen die Eliteschüler des Fußballs erwartungsgemäß sehr gute Notenleistungen im Vergleich zu den RegelschülerInnen mit guten Sportnoten (Gruppe: $p=, 002 * * / \eta^{2}=, 092$ ).

\section{Personale Ressourcen}

In Betrachtung der personalen Ressourcen zeigen sich für alle SchülerInnen sehr positiv ausgeprägte Selbsteinschätzungen im Bereich des Selbstwertgefühls sowie des schulischen und sportlichen Selbstkonzepts. Die Fußballer besitzen dabei sowohl ein höheres Selbstwertgefühl (Gruppe: $p \leq, 001 * * * / \eta^{2}=, 144$ ), als auch positivere Selbsteinschätzungen im sportlichen Selbstkonzept (Gruppe: $p=, 016^{*} / \eta^{2}=, 052$ ). Das Selbstwertgefühl unterliegt von der fünften bis zur siebten Klassenstufe einem negativen Entwicklungstrend (MZP: $\left.p=, 026 * / \eta^{2}=, 033\right)$.

\section{Soziale Ressourcen}

Auch die Ergebnisse zu den sozialen Ressourcen belegen sowohl für die Eliteschüler des Fußballs als auch für die RegelschülerInnen sehr gute soziale Beziehungen zu den wichtigsten Bezugsgruppen. Das soziale Selbstkonzept zur Schulklasse unterliegt für alle SchülerInnen einem positiven Messzeitpunkteffekt über den Untersuchungszeitraum (MZP: $\left.p=\leq, 001 * * * / \eta^{2}=, 134\right)$, wonach Gleichaltrige mit zunehmendem Alter eine bedeutendere Rolle im Leben der Heranwachsenden spielen. Im Vergleich von Fußballern und RegelschülerInnen konstatieren sich die Fußballer weiter positivere Beziehungen sowohl zu ihren Eltern (Gruppe: $p=, 016^{*} / \eta^{2}=, 052$ ) als auch zu ihrem Klassenlehrer bzw. zu ihrer Klassenlehrerin (Gruppe: $p=, 004 * * / \eta^{2}=, 073$ ). 
Tabelle 1: Ergebnisse der dreijährigen Längsschnittuntersuchung - Unterschiedsprüfung zwischen Eliteschülern des Fußballs (EdF) und RegelschülerInnen (Reg.) von der fünften bis zur siebten Klassenstufe (ANOVA MW)

\begin{tabular}{|c|c|c|c|c|c|c|c|c|c|}
\hline & & & & t3 & Ergebnisse de & er ANOVA & MW & & \\
\hline & & $M(S D)$ & $M(S D)$ & $M(S D)$ & Varianzquelle & F-Wert & df & $\begin{array}{c}\text { Sig. } \\
\text { (p-Wert) }\end{array}$ & $\eta^{2}$ \\
\hline Freizeitaktivitä & ât (Std./W.) & & & & & & & & \\
\hline Gesamt & $\mathrm{EdF}(\mathrm{N}=23)$ & $5,75(2,86)$ & $8,57(2,39)$ & $9,55(3,81)$ & MZP & 10,736 & 2 & $\leq, 001 * * *$ & ,100 \\
\hline & Reg. $(\mathrm{N}=78)$ & $4,80(3,53)$ & $5,90(4,97)$ & $5,61(4,84)$ & MZP x Gruppe & 4,123 & 2 &, 018 * & ,041 \\
\hline & & & & & Gruppe & 10,236 & 1 &, $002^{\star *}$ & ,095 \\
\hline Sport & $\mathrm{EdF}(\mathrm{N}=23)$ & $5,23(2,69)$ & $8,15(2,50)$ & $9,20(3,77)$ & MZP & 16,124 & 2 & $\leq, 001$ *** & ,143 \\
\hline & Reg. $(\mathrm{N}=78)$ & $2,96(2,68)$ & $3,28(3,34)$ & $3,85(3,06)$ & MZP x Gruppe & 7,271 & 2 &, $001^{\star * *}$ & ,070 \\
\hline & & & & & Gruppe & 46,356 & 1 & 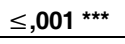 & ,323 \\
\hline Noten & & & & & & & & & \\
\hline Mathematik & $\mathrm{EdF}(\mathrm{N}=23)$ & $2,52(, 846)$ & $3,00(, 739)$ & $3,13(, 694)$ & MZP & 20,032 & 2 & $\leq, 001 * * *$ & ,159 \\
\hline & Reg. $(\mathrm{N}=85)$ & $2,58(, 968)$ & $3,14(, 888)$ & $3,33(, 944)$ & MZP x Gruppe & ,208 & 2 & ,804 & ,002 \\
\hline & & & & & Gruppe &, 619 & 1 &, 433 & ,006 \\
\hline Deutsch & $\mathrm{EdF}(\mathrm{N}=23)$ & $2,91(, 733)$ & $2,96(, 706)$ & $2,96(, 475)$ & MZP & 1,616 & 1,738 & ,201 &, 015 \\
\hline & Reg. $(\mathrm{N}=84)$ & $2,81(1,012)$ & $3,06(, 700)$ & $3,10(, 754)$ & MZP x Gruppe & ,847 & 1,738 & ,430 & ,008 \\
\hline & & & & & Gruppe & _. & 1 & ,756 & ,001 \\
\hline Sport & $\mathrm{EdF}(\mathrm{N}=23)$ & $1,22(, 518)$ & $1,39(, 583)$ & $1,17(, 388)$ & MZP & 2,058 & 2 & , 130 &, 019 \\
\hline & Reg. $(\mathrm{N}=83)$ & $1,65(, 706)$ & $1,82(, 814)$ & $1,77(, 941)$ & MZP x Gruppe &, 590 & 2 &, 555 & ,006 \\
\hline & & & & & Gruppe & 10,564 & 1 &, $002^{\star *}$ &, 092 \\
\hline Personale Res & ssourcen & & & & & & & & \\
\hline Selbstwert- & $\mathrm{EdF}(\mathrm{N}=23)$ & $3,76(, 255)$ & $3,74(, 306)$ & $3,66(, 374)$ & MZP & 3,724 & 2 & ,026* & ,033 \\
\hline gefühl & Reg. $(\mathrm{N}=88)$ & $3,42(, 571)$ & $3,34(, 629)$ & $3,16(, 678)$ & MZP x Gruppe & 750 & 2 & ,472 & ,007 \\
\hline & & & & & Gruppe & 14,042 & 1 & $\leq, 001^{\star \star *}$ & ,114 \\
\hline Schulisches & $\mathrm{EdF}(\mathrm{N}=23)$ & $3,25(, 589)$ & $3,11(, 505)$ & $2,91(, 696)$ & MZP & 2,806 & 1,693 & ,072 & 025 \\
\hline Selbstkonzept & Reg. $(\mathrm{N}=88)$ & $2,98(, 695)$ & $2,94(, 614)$ & $2,92(, 643)$ & MZP x Gruppe & 1,441 & 1,693 & ,240 &, 013 \\
\hline & & & & & Gruppe & 1,510 & 1 & ,222 & ,014 \\
\hline Sportliches & $\mathrm{EdF}(\mathrm{N}=23)$ & $3,46(, 424)$ & $3,32(, 449)$ & $3,56(, 400)$ & MZP & 1,573 & 2 &, 210 &, 014 \\
\hline Selbstkonzept & Reg. $(\mathrm{N}=88)$ & $3,13(, 683)$ & $3,14(, 695)$ & $3,15(, 646)$ & MZP x Gruppe & 1,855 & 2 & ,161 &, 017 \\
\hline & & & & & Gruppe & 5,957 & 1 &, $016^{\star}$ &, 052 \\
\hline Soziale Resso & urcen & & & & & & & & \\
\hline Fürsorglichkeit & $\mathrm{EdF}(\mathrm{N}=23)$ & $3,80(, 337)$ & $3,82(, 315)$ & $3,82(, 357)$ & MZP & ,098 & 1,784 & ,887 & ,001 \\
\hline Eltern & Reg. $(\mathrm{N}=88)$ & $3,60(, 525)$ & $3,57(, 608)$ & $3,52(, 612)$ & MZP x Gruppe & ,290 & 1,784 & ,723 & ,003 \\
\hline & & & & & Gruppe & 6,009 & 1 & ,016* & ,052 \\
\hline Fürsorglichkeit & $\mathrm{EdF}(\mathrm{N}=23)$ & $3,53(, 403)$ & $3,59(, 577)$ & $3,48(, 635)$ & MZP &, 621 & 2 &, 534 &, 006 \\
\hline Klassenlehrer & Reg. $(\mathrm{N}=88)$ & $3,25(, 663)$ & $2,96(, 907)$ & $3,22(, 846)$ & MZP x Gruppe & 1,976 & 2 & ,143 & ,018 \\
\hline & & & & & Gruppe & 8,579 & 1 &, $004^{\star *}$ & ,073 \\
\hline Soziales & $\mathrm{EdF}(\mathrm{N}=23)$ & $2,77(, 727)$ & $3,05(, 682)$ & $3,37(, 738)$ & MZP & 16,695 & 2 & $\leq, 001$ *** &, 134 \\
\hline Selbstkonzept & Reg. $(\mathrm{N}=88)$ & $2,66(, 754)$ & $2,97(, 708)$ & $3,08(, 629)$ & MZP x Gruppe & ,791 & 2 & ,454 & ,007 \\
\hline Schulklasse & & & & & Gruppe & 1,616 & 1 & ,206 &, 015 \\
\hline
\end{tabular}

Legende: $\mathrm{EdF}=$ Eliteschüler des Fußballs; Reg. = RegelschülerInnen; MZP = Messzeitpunkt; Gruppe = Eliteschüler des Fußballs vs. RegelschülerInnen; Signifikanzniveau: hoch signifikant (***), wenn $p \leq$ , 001 , sehr signifikant $(* *)$, wenn $p \leq, 01$ und signifikant $(*)$, wenn $p \leq, 05$; signifikante Ergebnisse fettgedruckt 


\section{Diskussion}

Die Ergebnisse der vorliegenden Untersuchung lassen sich hervorragend in den existierenden Forschungsstand einordnen. Die Eliteschüler des Fußballs weisen unter Berücksichtigung der Noten sowie der sozialen und personalen Ressourcen keinerlei Entwicklungsnachteile im Vergleich zu RegelschülerInnen auf, trotz zunehmender sportlicher Anforderungen in der Freizeit (Brettschneider/Klimek 2009). Entsprechend gängiger Forschungsbefunde besitzen die Eliteschüler des Fußballs höhere personale Ressourcen im Bereich des allgemeinen Selbstwertgefühls und sportlichen Selbstkonzepts (Beckmann/Szymanski/Elbe/Ehrlenspiel 2006; Breithecker 2016; Gerlach/Brettschneider 2013; Heim 2002) sowie höhere soziale Ressourcen unter Berücksichtigung der Eltern und des Klassenlehrers bzw. der Klassenlehrerin (Beckmann/Szymanski/Elbe/Ehrlenspiel 2006; Brettschneider/Klimek 2009; Heim 2002). Weiter lassen sich analog zu entwicklungspsychologischen Forschungsbefunden für alle Heranwachsenden gleichermaßen sowohl ein negativer Entwicklungsverlauf für das allgemeine Selbstwertgefühl als auch ein positiver Trend für das soziale Selbstkonzept zur Schulklasse ausmachen (Harter 2012). Das Konzept der Eliteschule des Fußballs des DFB scheint in den unteren Jahrgangsstufen der weiterführenden Schule folglich gute Unterstützungsleistungen zu bieten, um die Doppelbelastung aus Schule und Leistungsfußball zu meistern. Allerdings gilt es abzuwarten, wie sich die erhobenen Parameter im weiteren Verlauf der Schulzeit in den höheren Jahrgangsstufen entwickeln. Für repräsentative Forschungsbefunde wäre es insgesamt wünschenswert, die Untersuchung auf weitere Eliteschulen des Fußballs auszudehnen und so einen konsequenten, prozessbegleitenden Beitrag zur Schulentwicklung an den Eliteschulen des Fußballs zu leisten. Dies wäre nicht zuletzt aufgrund der alle drei Jahre anstehenden Neuzertifizierung als Eliteschule des Fußballs durch den DFB sowie der darüber hinaus stattfindenden Zertifizierung der Nachwuchsleistungszentren durch die Deutsche Fußball Liga (DFL) für alle schulischen und sportlichen Akteure von besonderem Interesse.

\section{Anmerkungen}

1 Das Forschungsprojekt wird auch aktuell weiter fortgeführt und wurde im Jahr 2016 zusätzlich durch eine neue Kohorte der SchulanfängerInnen (5. Klasse) erweitert.

2 Die Längsschnittstichprobe besteht ausschließlich aus männlichen Eliteschülern des Fußballs.

\section{Literatur}

Beckmann, J./Elbe, A.-M./Szymanski, B./Ehrlenspiel, F. (2006): Chancen und Risiken: Vom Leben im Verbundsystem von Schule und Leistungssport. Psychologische, soziologische und sportliche Leistungsaspekte. - Köln.

Breithecker, J. (2016): Die NRW-Sportschule: Chronischer Stress und Selbstkonzeptentwicklung von Sportprofilklassenschülern. - Augsburg (unveröffentl. Dissertation).

Brettschneider, W.-D./Kleine, T. (2002): Jugendarbeit im Sportverein: Anspruch und Wirklichkeit. Eine Evaluationsstudie. - Schorndorf.

Brettschneider, W.-D./Klimek, G. (2009): Sportbetonte Schulen. Ein Königsweg zur Förderung sportlicher Talente? (2. Aufl.) - Aachen.

Deutscher Fußballbund (DFB) (2016): Der Weg zur Eliteschule des Fußballs. Online verfügbar unter http://www.dfb.de/news/detail/der-weg-zur-eliteschule-des-fussballs-126224/, Stand: 15.04.2017 
Deutscher Fußballbund (DFB) (o. D.): Eliteschulen des Fußballs. Leitfaden für die Ausbildung. Online verfügbar unter

http://www.lfvm-v.de/fileadmin/user_up load/dateien/talentfoerderung/Leitfaden_Eliteschule.pdf, Stand: 15.04 .2017

Faltermaier, T. (1987): Lebensereignisse und Alltag. Konzeption einer lebensweltlichen Forschungsperspektive und eine qualitative Studie über Belastungen und Bewältigungsstile von jungen Krankenschwestern. - München.

Gerlach, E./Brettschneider, W.-D. (2013): Aufwachsen mit Sport. Befunde einer 10-jährigen Längsschnittstudie zwischen Kindheit und Adoleszenz. - Aachen.

Güllich, A./Richartz, A. (2016): Leistungssport im Kindes- und Jugendalter - ein Update. sportunterricht, 65,2, S. $49-54$.

Harter, S. (2012): The construction of the self ( $2^{\text {nd }}$ ed.). - New York.

Heim, R. (2002): Jugendliche Sozialisation und Selbstkonzeptentwicklung im Hochleistungssport. Eine empirische Studie aus pädagogischer Perspektive. - Aachen.

Hemming, K. (2015): Freizeitaktivitäten, chronischer Stress und protektive Ressourcen. Längsschnittstudie zu hohen Leistungsanforderungen in Sport und Musik im Kindesalter. - Wiesbaden. https://doi.org/10.1007/978-3-658-10278-4

Kehne, M./Breithecker, J. (2017): NRW-Sportschule: Zur Vereinbarkeit von Schule und sportlicher Förderung. sportunterricht, 66, 1, S. 14-19.

Lazarus, R.S./Folkman, S. (1984): Stress, Appraisal, and Coping. - New York.

Richartz, A. (2000): Lebenswege von Leistungssportlern. Anforderungen und Bewältigungsprozesse der Adoleszenz. Eine qualitative Längsschnittstudie. - Aachen.

Richartz, A./Brettschneider, W.-D. (1996): Weltmeister werden und die Schule schaffen. Zur Doppelbelastung von Schule und Leistungstraining. - Schorndorf.

Richartz, A./Hoffmann, K./Sallen, J. (2009): Kinder im Leistungssport. Chronische Belastungen und protektive Ressourcen. - Schorndorf.

Schwarzer, R./Jerusalem, M. (2002): Das Konzept der Selbstwirksamkeit. In: Jerusalem, M./Hopf, D. (Hrsg.): Selbstwirksamkeit und Motivationsprozesse in Bildungsinstitutionen (S. 28-53). - Weinheim.

Wild, E./Möller, J. (Hrsg.) (2015): Pädagogische Psychologie (2. Aufl.). - Berlin. 